\title{
Solution to Tool Swept Volume of Multi-axis Milling Simulation
}

\author{
Yu Tianbiao ${ }^{1, a}$, Zhang Xuewei ${ }^{1, a}$, Liu Wenwen ${ }^{1, a}$, Dai Yuanxing ${ }^{1, a}$, Li Queping $^{2, b}$ \\ ${ }^{1}$ School of Mechanical and Automation, Northeastern University, Shenyang, China \\ ${ }^{2}$ Shenyang Blower Works Group Corporation, Shenyang, China \\ aemail: xuewzhang@163.com, bemail:332828953@126.com
}

Keywords: multi-axis milling; cutter swept volume; enveloping characteristic line; general cutter model

\begin{abstract}
The tool swept volume has been generally applied in multi-axis NC machining simulation and verification. For the characteristic and actual condition, a general cutter model was built, and based on that a new method to simulate the tool swept volume of multi-axis milling was represented. The geometric model of cutter is translated to tool sphere with Guass Mapping, and the tangency function of tool vector was obtained. Combining the tool sphere and tangent function above, the enveloping characteristic line of tool could be acquired. Then the tool swept volume model was built. This paper also solves the self intersection of tool swept volume. Finally the simulation model verifies the validity of this method.
\end{abstract}

\section{Introduction}

In recent years, with the rapid development of computer technology and manufacturing, computer-aided design has become an important symbol to measure national manufacturing competitiveness. As an important part of the computer-aided design, multi-axis NC machining simulation technology based on computer graphics has been widely used in complex surface machining, which is helpful to improve the efficiency and quality of processing, and reduce the production cycle. In NC machining simulation and verification [1], solving for the tool swept volume is a key technology, and then the Boolean operations of the blank and tool swept volume for simulating the material removal could be obtained, and finally the process of dynamic could be display. Tool swept volume is the sum of the geometric space occupied by the tool contour to scan the parent entity at a time along the spatial scanning trajectory. Tool swept volume visualization [2] and its analysis has become a research hotspot. At present, the tool swept volume calculation method comprises the envelope theory method [3], scanning differential equations [4], the Jacobian method [5], Minkowski and [6], implicit modeling method [7] and so on. Scanning differential equations method, the Jacobian method, the implicit function modeling method requires complex mathematical operations, and the operating speed is slower. It is more difficult to achieve in this way. Envelope theory is the most widely used. Recent research about swept profile calculations of envelope theory are often established on the basis of differential equations. It is not precise enough for acquiring the profile of swept volume, the overall simulation result is also affected. These methods can be divided into two categories in essence: the first method is the discrete modeling. We uses Boolean union operations to describe the tool entity at each discrete location in the scan path. Discrete modeling method is easy to achieve, but the simulation accuracy is low. The second method is the Solid Modeling. We extract the corresponding scanning profile in each time period in accordance with the direction of movement, when we scan the tool entity. Finally we create the envelope surface of the swept volume with scanning profile obtained.

This paper chooses the envelope theory as the theoretical basis. It presents the method which is based on solid modeling and Guass mapping. The method consists of the following sections: Firstly, a generic tool model is created. And the universal tool model with gauss map is presented. Then the appropriate tool scanning profile according to the direction of movement and the Guass mapping of tool model is got. Finally the tool swept volume is build by interpolation principle in the scan path. This method can be used to obtain accurate tool swept contour and ensure the continuity of the surface of the tool. And then the quality and efficiency of machining simulation could be improved 


\section{Geometric model of universal cutter}

To ensure the generality of the method presented in this paper, we introduced universal cutter model for describing the sweeping volume. The mobile frame is built on the universal cutter, and the model was generally determined by seven parameters, as is shown in Fig.1.

We assumed the local coordinate system for the tool motion is $O_{T}-X_{T} Y_{T} Z_{T}$, which is shown in Fig.2 The geometric definition of cutter model is the solid of revolution formed by rotating the cutter contour, and the geometric model of universal cutter is composed by the upper tapered surface, the transition arc surface and the lower tapered surface. Geometric parameter equation of general cutter is as follows:

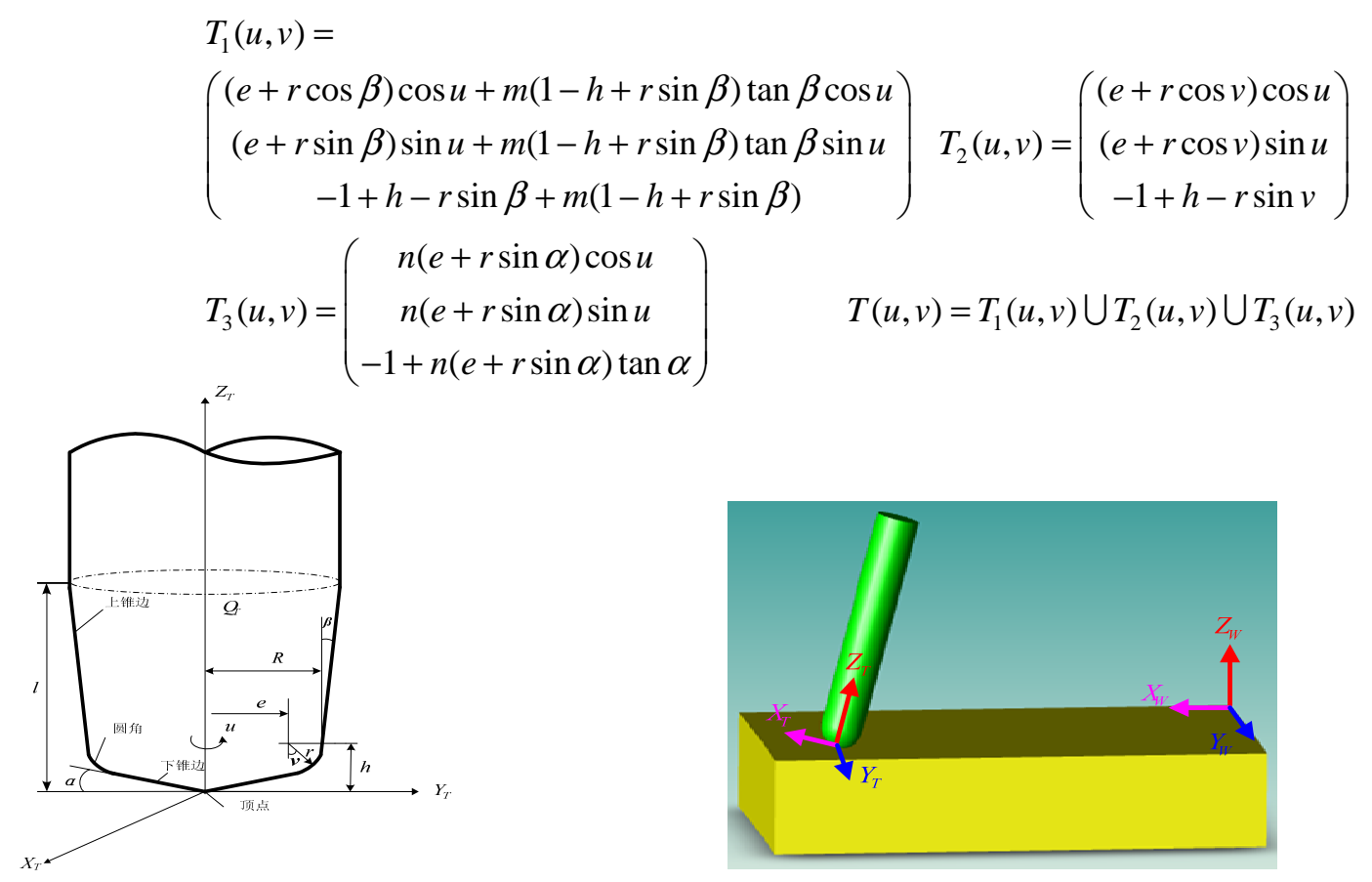

Fig.1.The model of univeral cutter Fig.2. The coordinate systems of cutter and workpiece

$R$ is the radius of the cutter; $e$ is the distance between cutter shaft and the center of tool bit corner, $r$ is radius of corner arc, $l$ is the height of the cutter, $h$ is the distance between tool bit and the center of corner, $\alpha$ is the angle between lower conical edge and the y-axis, $\beta$ is the angle between upper conical edge and the z-axis, $m \in[0,1], v \in[\alpha, 2 / \pi-\beta], u \in[0,2 \pi], T_{1}$ is upper conical surface, $\quad T_{2}$ is transition arc surface, $T_{3}$ is the lower conical surface.

Assuming the tool surface is smooth and regular surface patches, and the unit normal vectors of every point on the cutter surface:

$$
n(u, v)=\frac{T_{u} \times T_{v}}{\left|T_{u} \times T_{v}\right|}
$$

\section{Building cutter swept volume model based on cutter sphere}

In fact, building cutter swept volume is for solving the sweeping envelope surface of cutter. However, there are some theoretical difficulties and huge amount of calculation in the research of obtaining cutter envelope surface. Therefore, a relatively fast and accurate method for building models aimed at milling cutter swept volume is raised.

A. The Definition of cutter swept volume and its boundary

For five-axis milling, the local coordinate system of cutter along the continuous movement path is $O_{m}-X_{m} Y_{m} Z_{m}$. The cutter movement of five-axis machining can be transformed into translational and rotary motion with the origin of the cutter coordinate system.

Assuming that the tool movement path is $\delta(t), t$ is the time variable of the cutter movement, $A(t)$ is the orthogonal matrix which corresponds to the unit matrix $A(0), \mathbb{Z}=(x, y, z)$ is the point 
on the surface of cutter geometry model. The movement of cutter geometry model could be expressed as follows:

$$
S_{t}(\mathbb{Z})=\delta(t)+A(t) \cdot \mathbb{Z}
$$

According to the definition of cutter swept volume, the cutter swept volume which is formed along the cutter movement path is

$$
S V(t)=\bigcup\{S(t, \mathbb{Z}), t\}
$$

$t_{0}$ is the starting time of cutter movement, $t_{1}$ is the ending time of the cutter movement.

To obtain the envelope surface of cutter swept volume, the tangent function [8] is defined as follows:

$$
f=v\left(P_{i}\right) \cdot n\left(P_{i}\right)
$$

$P_{i}$ is the point on the surface of cutter, $n\left(P_{i}\right)$ is the normal vector on the surface of cutter, $v\left(P_{i}\right)$ is the velocity vector of $P_{i}$. The surface of cutter is divided into three parts by the tangent function:

(a) The front surface of cutter: $f=v\left(P_{i}\right) \bullet n\left(P_{i}\right)>0$

(b) The back surface of cutter: $f=v\left(P_{i}\right) \bullet n\left(P_{i}\right)<0$

(c) The envelope characteristic line of cutter:

$f=v\left(P_{i}\right) \bullet n\left(P_{i}\right)=0$, which is the dividing line of the front and back surface of the cutting tools geometry model.

Therefore, the cutter swept volume can also be presented as follows:

$$
\begin{aligned}
S V(t)= & S_{\text {back }}\left(t_{0}, \mathbb{Z}\right) \\
& \bigcup\left\{S_{\text {envelope }}(t, \mathbb{Z}), t_{0} \leq t \leq t_{1}\right\} \bigcup S_{\text {front }}\left(t_{1}, \mathbb{Z}\right)
\end{aligned}
$$

The cutter swept volume has three parts: the back surface of cutter $S_{\text {back }}\left(t_{0}, \mathbb{Z}\right)$ on the starting position of movement, the front surface of cutter $S_{\text {front }}\left(t_{1}, \mathbb{Z}\right)$ on the ending position of movement, and the envelope characteristic line of cutter $S_{\text {envelope }}(t, \mathbb{Z}), t \in\left[t_{0}, t_{1}\right]$ on other position of movement. The quality of machined workpiece depends on Boolean operation between the workpiece and cutter swept volume, and thus the envelope characteristic line of cutter has an important significance.

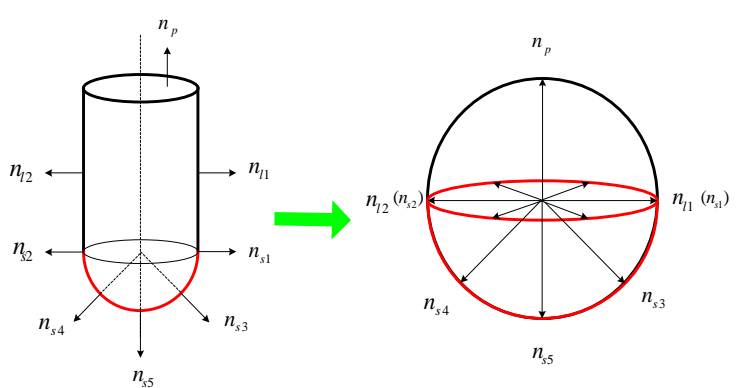

Fig.3. The cutter sphere based on Guass map characteristic line

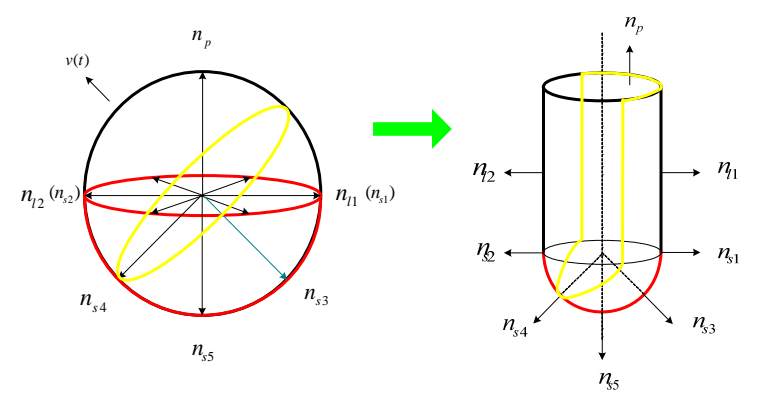

Fig.4. The solution to cutter envelope

\section{B. Gauss Map of Surface}

For the known surface $S: r(u, v), n(u, v)$ is the unit normal vector at any point of the surface $S$. The unit normal vector $n(u, v)$ is moved parallel to the origin, and the surface $S^{2}$ could be got by its terminal point. As $|n(u, v)|^{2}=1$, the surface $S^{2}$ is the part of the spherical surface $\rho^{2}=1$, and the map from surface to spherical surface is Guass.

Assuming that the tool geometry model of surface $T(u, v)$, the cutter surface unit normal vector $n(u, v)$ is obtained. Moving the unit normal vector of cutter surface to the origin parallel according to the define of Gauss map, the part of spherical surface could be obtained [9]. The sphere obtained is defined as the cutter sphere. 
Taking ball endmill as an example, as shown in Fig.3, the top surface on the ball mill is turned into the point of Gauss sphere, the cylindrical sphere is transformed into circle through center of Gauss sphere, and the hemispherical is transformed into hemisphere of Gauss sphere.

In the obtained cutter sphere, the front surface, the back surface and the envelope characteristic line of cutter could be obtained by the tangency function as shown in Fig.4. For the cutter swept volume, the acquisition of cutter envelope characteristic line could be transformed into tangent function between the unit normal vector and velocity of cutter.

Based on this method, the ball endmill swept volume under three-axis milling and five-axis milling are created in $\mathrm{C}++$ and OpenGL to valid the feasibility, as shown in Fig.5.
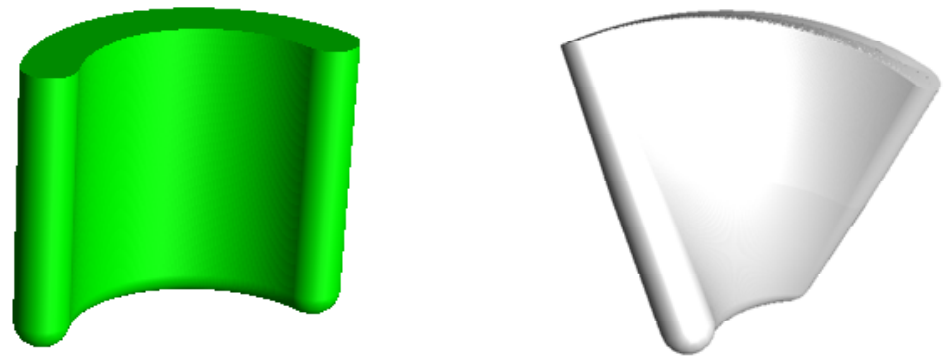

Fig.5. The model of ball endmill swept volum

\section{Conclusion}

This paper builds the general cutter model. As a prerequisite to generality and availability, the cutter swept volume is studied. Based on Guass map, the enveloping characteristic line can be obtained by the cutter sphere, which ensures the accuracy and quickness. Finally the correctness of the method presented is verified through the simulation results.

\section{References}

[1] L. Bert, P. Denys. Five-axis milling tool path generation with dynamic step-over calculation based on integrated material removal simulation[J], CIRP Annuals-Maufacturing Technology, 2012(1)139-142.

[2] S. Liang. Research and implementation of key technologies of solid-based simulation of NC machining[C]. Nanjing: Nanjing University of Aeronautics and Astronautics, 2005.

[3] W.P.Wang, K.K.Wang. Geometric modeling for swept volume of moving solids[J]. IEEE Computer Graphics and Applications, 1986(6) 8-17.

[4] D.Blackmore, M.C.Leu. A differential equation approach to swept volumes[J]. IEEE Comput.Soc, 1990(3)143-149.

[5]M.K.Abdel, H.J.Yeh, S.Othman. Swept volumes: void and boundary identification[J]. Computer-Aided Design, 1998(30)1009-1018.

[6] S. Gaemers, C.J. Elsevier, A. Bax. Offsets, sweeps, and minkowski sums[J]. Computer-Aided Design, 1999(31)163-163.

[7] W.J.Schmidt, W.E.Lorensen, S.Linthicum. Implicit modeling of swept surfaces and volumes[J]. Los Alamitos:IEEE Comput.Soc[J], 1994(5):40-45.

[8]E. Kreyszig. Differential geometry. New York: Dover Publications, 1991.

[9]W.L.Seok, N.Andreas. Virtual workpiece: workpiece representation for material removal process[J], Computer-Aided Design, 2011(58)443-463. 